\title{
Application effect of high-quality nursing on patients with coronary heart disease.
}

\author{
Ying Shen ${ }^{1 \#}$, Songqi Guo"\#, Yingli Hou ${ }^{3 *}$ \\ ${ }^{1}$ Department of Gegiatrics, International Outpatient Department, Union Hospital, Tongji Medical College, Huazhong \\ University of Science and Technology, Wuhan, Hubei, PR China \\ ${ }^{2}$ Cardiac Care Unit, the First Affiliated Hospital, Harbin Medical University, Harbin, Heilongjiang, PR China \\ ${ }^{3}$ Department of Cardiology, Shaanxi Second People's Hospital, Xi'an, Shaanxi, PR China \\ \#These authors contributed equally to this paper
}

\begin{abstract}
Objective: This study aimed to identify the application effect of high-quality nursing on patients with coronary heart disease.

Methods: We recruited 112 patients with coronary heart disease who were admitted to our hospital over the period of December 2015 to June 2017. We randomly assigned 56 patients to the control group and 56 patients to the experimental group. The control group received conventional nursing, and the control group received high-quality nursing. Emotional changes, nursing satisfaction, compliance, and living quality of two groups were observed and compared.

Results: The two groups reported lower anxiety scores after nursing intervention than before nursing intervention. The anxiety score of the experimental group was significantly lower than that of the control group $(\mathbf{P}<\mathbf{0 . 0 5})$. The experimental group reported significantly higher nursing satisfaction than the control group $(\mathbf{P}<\mathbf{0 . 0 5})$. Compared with the control group, the experimental exhibited better compliance behaviors, such as taking medicines in accordance with medical advice, maintaining a reasonable diet, performing appropriate exercise, and emotional stability $(\mathrm{P}<0.05)$. Compared with the control group, the experimental group had better living quality, in terms of physiological function, social function, rational drug use, standard diet, and self-monitoring $(P<0.05)$.

Conclusions: High-quality nursing changes unhealthy emotions, increases compliance behaviors and nursing satisfaction, and improves living quality of patients with coronary heart disease. Therefore, high-quality nursing is worthy of clinical references and applications.
\end{abstract}

Keywords: High-quality nursing, Coronary heart disease, Application effect.

Accepted on December 26, 2017

\section{Introduction}

Coronary Heart Disease (CHD) is a common cardiovascular disease. It is associated with high morbidity rates among the elderly [1]. CHD morbidity rates have continuously increased with the aging population. The delayed or improper treatment of CHD increases the occurrence and fatality rates of major adverse cardiovascular events [2]. Safe, high-quality, and systematic nursing service provided in accordance with the recommended treatment can effectively improve disease conditions, prognosis, long-term survival, and life quality of patients with CHD [3]. Unlike conventional nursing, which focuses solely on managing the disease, high-quality nursing focuses on patients to improve therapeutic effect and survival quality [4]. In this study, we recruited 112 patients with coronary heart disease who were admitted to our hospital over the period of December 2015 to June 2017. Results are introduced in the following sections.

\section{Information and Methods}

\section{General information}

We recruited 112 patients with coronary heart disease and who were admitted to our hospital over the period of December 2015 to June 2017. All patients conformed to the WHO clinical diagnostic standards for CHD and were diagnosed through coronary arteriography. Patients with cognitive disorders, cardiac function $<2$ level, mental diseases, and acute myocardial infarction were excluded. The recruited patients were randomly divided into the control and experimental groups. Each group contained 56 cases. The control group included 32 males and 24 females aged 58-80 with a mean age 
of $68.6 \pm 8.2$. The control group included 35 cases of stenocardia, six cases of myocardial infarction, and 24 cases of asymptomatic myocardial ischemia. The experimental group included 31 males and 25 females aged 59-81 with a mean age of $69.5 \pm 8.1$. The experimental group included 34 cases of stenocardia, eight cases of myocardial infarction, and 14 cases of asymptomatic myocardial ischemia. The general information of the two groups was not significantly different $(\mathrm{P}>0.05)$. Thus, the two groups were comparable.

\section{Method}

The control group received conventional nursing, or basic nursing, for CHD. Meanwhile, disease changes, vital sign changes, and adverse drug reactions were monitored. The experimental group received high-quality nursing, which encompassed the following aspects.

Basic nursing service upon hospital admission: Most patients with CHD feel nervous and fear the strange hospital environment and medical care personnel. Nursing staff should smile constantly when receiving patients and familiarizing them with the hospital environment and staff because patients can perceive the respect and care of medical staff. Emotional changes should be strictly monitored. Nursing staff should focus on and provide specific solutions to the psychological problems of patients, as well as promote the compliance behavior of patients. Nurses should make ward rounds every 15-40 min during venous transfusion and should inform patients about the specific and common untoward effects of the administered drugs. Patients should be instructed to follow medical advice strictly to prevent burdening the liver and kidney through erroneous or improper medication. The types and dosage of medicines should be decreased appropriately when warranted.

Mental nursing and behavioral intervention should be performed because CHD is a psychosomatic disease. The influences of social and psychological factors on the diagnosis and treatment behavior of patients should be emphasized. Nursing staff should understand the daily activities and personal characteristics of patients and their psychological conditions. Corresponding comfort and pertinent psychological nursing measures should be formulated. Nursing staff should provide patients and their family members with information about the preventive strategies, common causes, and complications of CHD; help patients comprehend the negative relationship between unhealthy emotions and disease rehabilitation; encourage patients to maintain a positive and optimistic psychological attitude; encourage and support patient-nurse communication; and present cases of other patients with CHD who have successfully recovered to help patients feel confident about their recovery and encourage their positive cooperation with treatment and nursing.

Guidance on safe nursing: Nursing staff should perform morning and evening nursing work; maintain the cleanliness of ward beds and environment; prevent trash accumulation in inpatient wards; assist life-support-independent patients in turning over and eating; strengthen the mouth and skin care of life-support-dependent patients to prevent pressure sores; emphasize the importance of overall nursing service; regularly attend business knowledge learning and training; enhance nursing service consciousness; update nursing concepts and ideas; actively implement responsibility systems; and ensure nursing safety.

Diet intervention: Nursing staff should ask the patients to eat digestible foods with low amounts of spices and fats; control their salt intake; take $\mathrm{Ca}, \mathrm{K}$, and high-quality protein supplements; consume high amounts of vegetables and fruits to prevent constipation; and correct improper dietary habits.

Rehabilitation guidance and discharge instruction: Nursing staff should support and encourage patients to perform off-bed activities as early as possible during hospitalization in accordance with basic hygienic knowledge education and psychological intervention; formulate a long-term rehabilitation and treatment program; establish confidence in disease recovery and positive life outlook; prevent patients from drinking strong tea and coffee; and determine long-term management methods and skills. After discharge from the hospital, the nursing staff should perform telephone follow-ups and visits and family follow-up visit if necessary. The importance of regular reexamination should be stressed to the patients and their family members. Nursing staff should advise patients to return to the hospital for any anomaly.

\section{Observation indexes}

Emotional changes: Changes in the emotions of patients before nursing intervention and 3 months after nursing intervention were evaluated using the self-rating anxiety scale. High scores indicate serious anxiety.

Compliance behavior: Compliance behavior of patients was assessed 3 months after nursing intervention. Compliance behaviors mainly included taking medicines in accordance with medical advice, performing appropriate exercise, consuming a reasonable diet, and emotional stability.

Nursing satisfaction was evaluated using a nursing satisfaction questionnaire designed by the hospital. The questionnaire had a total score of 100 and was divided into three levels: very satisfactory ( $>80$ scores), satisfactory (60-80 scores), and unsatisfactory ( $<60$ scores).

Living quality was evaluated by SF-36 scale and included standard diet, social function, self-monitoring, physiological function, and rational drug use. Low scores represented the poor living quality of patients.

\section{Statistical analysis}

All data were analysed by SPSS122.0 statistical software. The measurement data of emotional changes and living quality were expressed as $(\overline{\mathrm{x}} \pm \mathrm{s})$ and were examined through t-test. Enumeration data of nursing satisfaction and compliance behavior were expressed by $\%$ and were examined through $\chi^{2}$ test. $\mathrm{P}<0.05$ indicated statistically significant differences between the two groups. 


\section{Results}

\section{Emotional changes before and after nursing intervention}

Before nursing intervention, the anxiety score of the control group was similar to that of the experimental group $(\mathrm{P}>0.05)$. After nursing intervention, the anxiety score of the experimental group was significantly lower than that of the control group $(\mathrm{P}<0.05)$ (Table 1).

Table 1. Comparison of emotional changes of the two groups before and after nursing intervention ( $\bar{x} \pm s$, scores).

\begin{tabular}{llll}
\hline Group & Cases & Before & After \\
\hline Control group & 56 & $61.8 \pm 5.2$ & $57.8 \pm 5.1$ \\
\hline Experimental group & 56 & $62.2 \pm 5.4$ & $40.1 \pm 4.6$ \\
\hline $\mathrm{t}$ & & 0.3992 & 19.2856 \\
\hline $\mathrm{P}$ & 0.6905 & 0.0000 \\
\hline
\end{tabular}

\section{Nursing satisfaction}

The control group expressed significantly lower nursing satisfaction than the experimental group $(\mathrm{P}<0.05)$ (Table 2).

Table 2. Comparison of nursing satisfaction between the two groups ( $(\%))$.

\begin{tabular}{llllll}
\hline Group & Cases & Very satisfactory & Satisfactory & Unsatisfactory & Degree of satisfaction \\
\hline Control & 56 & $27(48.2)$ & $21(37.5)$ & $7(12.5)$ & $48(85.7)$ \\
\hline Experimental & 56 & $35(62.5)$ & $20(35.7)$ & $1(1.8)$ & $55(98.2)$ \\
\hline$X^{2}$ & & & & 4.8462 & 0.0277 \\
\hline$P$ & & & & & \\
\hline
\end{tabular}

\section{Compliance behavior}

Compared with the control group, the experimental group reported better compliance behaviors, such as performing appropriate exercise, maintaining a reasonable diet, emotional stability, and taking medicines in accordance with medical advice $(\mathrm{P}<0.05)$ (Table 3$)$.

Table 3. Comparison of compliance behaviors between the two groups (n(\%)).

\begin{tabular}{|c|c|c|c|c|c|}
\hline Group & Cases & Emotional stability & Reasonable diet & Appropriate exercise & $\begin{array}{l}\text { Taking medicines in accordance } \\
\text { with medical advice }\end{array}$ \\
\hline Control & 56 & $47(83.9)$ & $48(85.7)$ & $46(82.1)$ & $50(89.3)$ \\
\hline Experimental & 56 & $54(96.4)$ & $55(98.2)$ & $53(94.6)$ & $56(100.0)$ \\
\hline$x^{2}$ & & 4.9297 & 5.9202 & 4.2642 & 6.3396 \\
\hline$P$ & & 0.0262 & 0.0150 & 0.0389 & 0.0118 \\
\hline
\end{tabular}

\section{Living quality}

The control group presented significantly lower living quality scores for physiological function, social function, rational drug use, standard diet, and self-monitoring than the experimental group $(\mathrm{P}<0.05)$ (Table 4$)$.

Table 4. Comparison of living quality between the two groups ( $\bar{x} \pm s$, scores).

\begin{tabular}{|c|c|c|c|c|c|c|}
\hline Group & Cases & Self-monitoring & Standard diet & Rational drug use & Social function & Physiological function \\
\hline Control & 56 & $54.9 \pm 4.5$ & $42.6 \pm 7.2$ & $45.7 \pm 7.1$ & $64.8 \pm 7.5$ & $77.8 \pm 8.1$ \\
\hline Experimental & 56 & $68.5 \pm 5.7$ & $73.8 \pm 9.2$ & $71.5 \pm 8.2$ & $79.6 \pm 9.2$ & $83.2 \pm 9.7$ \\
\hline $\mathrm{t}$ & & 14.0140 & 19.9854 & 17.7999 & 9.3307 & 3.1976 \\
\hline$P$ & & 0.0000 & 0.0000 & 0.0000 & 0.0000 & 0.0018 \\
\hline
\end{tabular}

\section{Discussion}

CHD is a common internal disease that threatens the physical and physiological health and life safety of patients. Requirements of patients for clinical nursing quality have increased given that their self-protection awareness has increased with recent social and economic developments $[5,6]$. Nursing quality determines the therapeutic effect and prognosis of patients and also affects the long-term development of the 
hospital. High-quality nursing, which is a novel nursing concept and scheme, provides mental, social, and psychological nursing guidance based on disease nursing $[7,8]$. Through safety nursing, psychological guidance, and diet intervention, high-quality nursing promotes the positive and optimistic attitudes of patients toward recovery and effectively helps avoid high-risk factors and prevent accidents, thus accelerating the rehabilitation of patients and improving nursing service quality and the patients' prognosis $[9,10]$. In contrast to conventional nursing, high-quality nursing requires nursing staff to master theoretical knowledge and practical skills by following the system of three-inspections and seven verifications to enhance the sense of trust and safety of patients and their family members. High-quality nursing also requires strict adherence to disinfection and isolation protocol, such as hand-washing and disinfection during nursing services, to prevent contamination and prevent cross-infection.

\section{Conclusion}

In this study, the experimental group achieved significantly lower anxiety scores and significantly higher living quality, compliance behavior, and nursing satisfaction than the control group $(\mathrm{P}<0.05)$. High-quality nursing improves the unhealthy emotions, compliance behavior, nursing satisfaction, and living quality of patients with CHD. Thus, it is worthy of clinical applications.

\section{Acknowledgments}

This work was supported by grants from the Heilongjiang Provincial Health Department Project (2012-546).

\section{References}

1. Dweck MR, Williams MC, Moss AJ, Newby DE, Fayad ZA. Computed tomography and cardiac magnetic resonance in ischemic heart disease. J Am Coll Cardiol 2016; 68: 2201-2216.

2. Min A. A case report of postpartum hemolytic uremic syndrome. Biomed Res India 2017; 28: 3808-3810.
3. Carnethon MR, Biggs ML, Barzilay J, Kuller LH, Mozaffarian D, Mukamal K, Smith NL, Siscovick D. Diabetes and coronary heart disease as risk factors for mortality in older adults. Am J Med 2010; 123: 556.e1-556.e9.

4. Li YJ, Li YM, Chen H, Sun WF. Possibility of bakuchiolinduced diseases through inhibition of specific drugmetabolizing enzymes (DMEs). Lat Am J Pharm 2013; 32: 924-926.

5. Infante T, Forte E, Schiano C, Cavaliere C, Tedeschi C, Soricelli A, Salvatore M, Napoli C. An integrated approach to coronary heart disease diagnosis and clinical management. Am J Transl Res 2017; 9: 3148-3166.

6. Yao C, Huang X, Li L. Perindopril improves cardiac function but not diaphragmatic fatigue in rats with chronic heart failure. Lat Am J Pharm 2015; 34: 30-35.

7. Shahoumian TA, Phillips BR, Backus LI. Cigarette smoking, reduction and quit attempts: prevalence among veterans with coronary heart disease. Prev Chronic Dis 2016; 13: 41.

8. Eriksson JG, Kajantie E, Thornburg KL, Osmond C, Barker DJP. Mothers body size and placental size predict coronary heart disease in men. Eur Heart J 2011; 32: 2297-2303.

9. Zhang J, Lu Q. Clinical efficacy of ultrafiltration in the treatment of acute decompensated heart failure with diuretic resistance. Biomed Res India 2017; 28: 6155-6159.

10. Hickey N, Maurer B, Mulcahy R, NcGilvray JW. Arcus senilis: its relation to certain attributes and risk factors in patients with coronary heart disease. Br Heart J 1970; 32: 449-452.

\section{*Correspondence to}

Yingli Hou

Department of Cardiology

Shaanxi Second People's Hospital

PR China 